\title{
The design of a homocysteine fluorescent probe based on Rhodamine $B$ and its responsiveness in the serum of cerebral infarction patients
}

\author{
XIUBAO $\mathrm{ZHAO}^{1 *}, \mathrm{XU} \mathrm{LI}^{2 *}, \mathrm{YING} \mathrm{MA}^{3 *}$ and $\mathrm{YU} \mathrm{ZHU}^{2}$ \\ ${ }^{1}$ Department of Intensive Care Unit, Tianjin First Central Hospital, Tianjin 300192; \\ ${ }^{2}$ Department of Clinical Laboratory, Tianjin Huanhu Hospital, Tianjin 300350; \\ ${ }^{3}$ Tianjin Key Laboratory on Technologies Enabling Development of Clinical Therapeutics and \\ Diagnostics (Theranostics), School of Pharmacy, Tianjin Medical University, Tianjin 300070, P.R. China
}

Received November 3, 2018; Accepted May 16, 2019

DOI: $10.3892 /$ etm.2019.7833

\begin{abstract}
High levels of homocysteine (Hcy) is closely associated with the onset of cerebral infarction. The present study aimed to synthesize a novel Hcy probe based on Rhodamine B, named S1-4, a new compound that has not been previously reported. This probe exhibited good linear range under physiological fluid viscosity and $\mathrm{pH}$; it has good selectivity for Hcy, and is able to avoid interference from other amino acids and metal ions. This probe can effectively measure the level of Hcy in the blood sera of healthy people and in patients with transient cerebral ischemia and cerebral infarction. However, satisfactory specificity and sensitivity to Hcy was not achieved according to receiver operating characteristic curve analysis. Overall, results from the present study suggested that following further optimization, this probe may be potentially applied in the diagnosis of cerebral infarction.
\end{abstract}

\section{Introduction}

Cerebral infarction, also known as acute ischemic stroke, is a common acute cerebrovascular disease that is harmful to the health of the elderly and the young (1). It is the characterized by high rates of recurrence (26\% within 5 years and $39 \%$ within 10 years), disability (19\% of patients within 3 months and $38 \%$ within 12 months) and mortality $(114.8 / 100,000)$ in China $(2,3)$,

Correspondence to: $\mathrm{Dr} \mathrm{Yu}$ Zhu, Department of Clinical Laboratory, Tianjin Huanhu Hospital, 6 Jizhao Road, Tianjin 300350, P.R. China

E-mail: zhuyutj@126.com

Dr Xiubao Zhao, Department of Intensive Care Unit, Tianjin First Center Hospital, 24 Fukang Road, Tianjin 300192, P.R. China

E-mail: zobby326@163.com

*Contributed equally

Key words: homocysteine, homocysteine probe, cerebral infarction which seriously affects the quality of life of patients, as well as causing substantial emotional and economic burden to both the patients and their families. Developed countries worldwide, including China, have a rapidly aging society; therefore, effective detection techniques for the early screening, risk prediction, diagnosis and prognosis of cerebral infarction are urgently needed $(3,4)$. Clinical and epidemiological data have confirmed that homocysteine (Hcy) is an important risk factor for cardiovascular diseases and is closely associated with the pathogenesis of cerebral infarction (5). The level of Hcy in the plasma of patients with cerebral infarction is significantly higher compared with that of the healthy population $(6,7)$. Therefore, efficient methods of monitoring Hcy levels provides a potentially useful avenue for the early diagnosis, prevention and treatment, as well as prognosis, of patients affected by cerebral infarction.

\section{Materials and methods}

Synthesis of the probe. The Hcy probe was synthesized according to the pathway illustrated in Fig. 1. Briefly, 4-formylbenzoic acid ('I'; $15.0 \mathrm{~g}, 0.1 \mathrm{M}$ ) was dissolved in $100 \mathrm{ml}$ of dichloromethane, and 5 drops of N,N-dimethylformamide was added as a catalyst. The reaction was cooled in an ice bath to approximately $0^{\circ} \mathrm{C}$, before oxalyl chloride ('a'; $19.05 \mathrm{~g}$, $0.15 \mathrm{M}$ ) was added dropwise. The reaction continued under rotary distillation at room temperature for $2 \mathrm{~h}$ to remove the solvent and excess oxalyl chloride. The 4-formylbenzoyl chloride ('II') obtained was used directly for the next step without purification. In a reaction flask, $50 \mathrm{ml}$ of ethylene glycol ('b') was added and cooled to approximately $0^{\circ} \mathrm{C}$ in an ice bath. 4-Formylbenzoyl chloride was gradually added to the ethylene glycol, and the reaction was continued under rotary distillation at room temperature for $2 \mathrm{~h}$ to remove excess ethylene glycol. Crude 2-hydroxyethyl 4-formylbenzoate ('III') was then purified by silica gel column chromatography and eluted using petroleum ether/ethyl acetate (2:1) as the eluent, and a colorless, waxy solid (15.7 g, 80.93\%) was obtained. Rhodamine B ('IV'; $2.39 \mathrm{~g}, 0.005 \mathrm{M}$ ) was dissolved in $30 \mathrm{ml}$ of dichloromethane before the sequential 
addition of 2-hydroxyethyl 4-formylbenzoate (1.16 g, $0.006 \mathrm{M})$, $\mathrm{N}$-(3-dimethylaminopropyl)-N'-ethylcarbodiimide hydrochloride $(0.96 \mathrm{~g}, 0.005 \mathrm{M})$ and 4 -(dimethylamino)pyridine $(0.12 \mathrm{~g}$, $0.001 \mathrm{M})$. The reaction was carried out at room temperature for $\sim 28 \mathrm{~h}$, and was monitored using thin-layer chromatography with dichloromethane/methanol (10:1) as the solvent. This reaction system was subjected to rotary distillation to remove the solvent dichloromethane. The compound was dissolved in $2 \mathrm{ml}$ dichloromethane and chromatography for purification was performed using a vitreous silica gel column (column diameter, $1.5 \mathrm{~cm}$; column length, $70 \mathrm{~cm}$; HuaSheng Chromatography, Jiangyin, China at a flow rate of $2 \mathrm{ml} / \mathrm{min}$ at room temperature. Dichloromethane/methanol $(50 \mathrm{ml} ; 10: 1)$ was used as the eluent. Finally, N-(6-(diethylamino)-9-(2-((2-(4-formylbenzoyloxy) ethoxy)carbonyl)phenyl)-3H-xanthen-3-ylidene)-N-ethylethanaminium chloride (' $\mathrm{V}$ ' also known as $\mathrm{S} 1-4 ; 0.471 \mathrm{~g}$, 14.4\%) was obtained, named thereafter as S1-4. All reagents mentioned above were purchased from Sigma-Aldrich (Merck KGaA). The compound structure ' $\mathrm{V}$ ' was dissolved by deuterium oxide and analyzed using ${ }^{1} \mathrm{H}$-Nuclear magnetic resonance ( ${ }^{1} \mathrm{H}-\mathrm{NMR}$; 400-MR DD2; $400 \mathrm{MHz}$; Agilent Technologies, Inc.) and an electron ionization-high resolution mass spectrometry (EI-HRMS; Micromass ${ }^{\circledR}$ GCT Premier ${ }^{\mathrm{TM}}$; Waters Corporation).

Determination of Hcy probe fluorescence linearity. The Hcy probe $(10 \mu \mathrm{M})$ was prepared in PBS (Beijing Solarbio Science $\&$ Technology Co., Ltd.). In a 96-well black microplate, 0,1 , 2, 5, 10, 20, 50 and $100 \mu \mathrm{M}$ Hcy (Beijing Solarbio Science $\&$ Technology Co., Ltd.), $100 \mu 1$ in human-based serum (cat. no. LUN5048; Randox Laboratories, Ltd.), was mixed with $100 \mu 1$ of the working solution of the Hcy probe. Following incubation at $37^{\circ} \mathrm{C}$ for $30 \mathrm{~min}$, fluorescence intensity was measured at the excitation wavelength of $280 \mathrm{~nm}$ and emission wavelength of $590 \mathrm{~nm}$ using a microplate reader (Thermo Fisher Scientific Inc.), from which a standard curve of concentration vs. fluorescence was generated.

Effect of fluid viscosity and $\mathrm{pH}$ on the response of the probe. The working solution of the probe $(10 \mu \mathrm{M})$ was prepared in PBS. In a 96-well black microplate, $100 \mu 1$ of $20 \mu \mathrm{M}$ Hcy with different fluid viscosities (glycerol/water=1.005-219 cp) were added to $100 \mu \mathrm{l}$ of the working solution of the probe. Following incubation at $37^{\circ} \mathrm{C}$ for $30 \mathrm{~min}$, fluorescence intensity was measured at an excitation wavelength of $280 \mathrm{~nm}$ and emission wavelength of $590 \mathrm{~nm}$ to analyze the effect of fluid viscosity on probe responsiveness using a microplate reader (Thermo Fisher Scientific Inc.). To measure the effect of $\mathrm{pH}$ on the response of the probe, buffers at $\mathrm{pH}$ 6.5-8.5 were prepared using $\mathrm{HCl}, \mathrm{NaOH}$ and PBS before $10 \mu \mathrm{M}$ of the probe was added to $20 \mu \mathrm{M} \mathrm{Hcy}$. Following incubation at $37^{\circ} \mathrm{C}$ for $30 \mathrm{~min}$, probe response was measured by the same method using a microplate reader (Thermo Fisher Scientific, Inc.).

Determination of probe selectivity. Considering the complex intracellular environment, various interfering substances, such as the main amino acids and other small molecules, were added to the probe mixture, including various amino acids derivatives [cysteine (Cys); Hcy; N-acetylcysteine; glutathione (GSH); arginine; asparagine; aspartic acid; $\beta$-alanine; dithiothreitol; glutamine; glutamic acid; glycine; histidine; isoleucine; L-alanine; leucine; lysine; methionine; phenylalanine; proline; serine; threonine; tryptophan; tyrosine; and valine; all $1 \mathrm{mM}$, metal ions [potassium $\left(\mathrm{K}^{+}\right)$; sodium $\left(\mathrm{Na}^{+}\right)$; calcium $\left(\mathrm{Ca}^{2+}\right)$; magnesium $\left(\mathrm{Mg}^{2+}\right)$; zinc $\left(\mathrm{Zn}^{2+}\right)$; aluminum $\left(\mathrm{Al}^{3+}\right)$; cadmium $\left(\mathrm{Cd}^{2+}\right)$; manganese $\left(\mathrm{Mn}^{2+}\right)$ ], and $\mathrm{Na}_{2} \mathrm{SO}_{3}$ and $\mathrm{H}_{2} \mathrm{~S}$ (made from $\mathrm{Na}_{2} \mathrm{~S} ; 1 \mathrm{mM}$ for both). The working solution of the probe $(10 \mu \mathrm{M})$ was prepared in PBS. In a 96-well black microplate, $100 \mu 1$ of each of the substances mentioned above were added to the working solution of the probe. Following incubation at $37^{\circ} \mathrm{C}$ for $30 \mathrm{~min}$, fluorescence intensity was measured at an excitation wavelength of $280 \mathrm{~nm}$ and emission wavelength of $590 \mathrm{~nm}$ to analyze probe selectivity in the presence of interfering substances. All reagents mentioned above were purchased from Beijing Solarbio Science \& Technology Co., Ltd.

Detection of Hcy in the serum using the probe and a biochemistry analyzer. A total of 50 patients with cerebral infarction (CI group) and 50 patients with transient cerebral ischemia (TIA group) diagnosed in Tianjin Huanhu Hospital (Tianjin, China) between May 2016 and November 2017 were enrolled into the present study. A total of 50 healthy volunteers were classified into the normal (Norm) group. All participants in the experiments were matched for age (TIA, 60.1 \pm 11.2 ; CI,

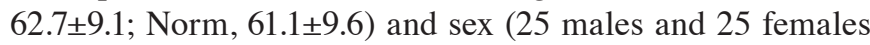
in each group). This study was approved by the medical ethics committee of Tianjin Huanhu Hospital, and followed the principles according to the Declaration of Helsinki; all patients provided informed consent before the experiments.

The inclusion criteria were as follows: i) Patients in the $\mathrm{CI}$ and TIA group were diagnosed within $24 \mathrm{~h}$ following the onset of symptoms according to the standard clinical criteria for CI and TIA, with supporting brain images produced either by computed tomography or magnetic resonance imaging and magnetic resonance angiography. The venous blood of Norm, $\mathrm{CI}$ and TIA groups were collected within $24 \mathrm{~h}$ of admission into hospital into an sterile and impermeable container before being frozen immediately at $-80^{\circ} \mathrm{C}$ after collection; and ii) asymptomatic patients undergoing physical examinations who did not suffer from previous or current cardiovascular and cerebrovascular diseases were enrolled into the healthy control (Norm) group. The exclusion criteria were as follows: i) Patients with intracerebral hemorrhage or other brain injuries, atherosclerosis, heart failure, respiratory failure, renal failure, severe liver dysfunction, malignant tumors, autoimmune disease, diabetes mellitus or any other metabolic diseases, intestinal disorders, or other malignant disease; and ii) admission $>24 \mathrm{~h}$ after stroke onset.

A total of $5 \mathrm{ml}$ venous blood was collected from patients in each of the groups and centrifuged at $300 \mathrm{xg}$ for $10 \mathrm{~min}$ at room temperature. Hcy content in the serum was measured using a Biochemistry Analyzer (Beckman Coulter, Inc.). Dithiothreitol (1 M, $5 \mu \mathrm{l}$; Sigma-Aldrich; Merck KGaA) was added to $95 \mu \mathrm{l}$ of the serum and incubated at $37^{\circ} \mathrm{C}$ for $30 \mathrm{~min}$, followed by the addition of the probe solution $(10 \mu \mathrm{M})$ and another round of incubation at $37^{\circ} \mathrm{C}$ for $30 \mathrm{~min}$. Fluorescence intensity was measured at the excitation wavelength of $280 \mathrm{~nm}$ and emission wavelength of $590 \mathrm{~nm}$ using a microplate reader (Thermo Fisher Scientific, Inc.). Using the fluorescence intensity data, a correlation and receiver operating characteristic 
A<smiles>[C-][N+](CC)=c1ccc2c(-c3ccccc3C(=O)O)c3ccc(N(CC)CC)cc3oc-2c1</smiles>

IV<smiles>O=C(Cl)C(=O)Cl</smiles><smiles>O=Cc1ccc(C(=O)Cl)cc1</smiles><smiles>OCCO</smiles>

b<smiles></smiles>

B

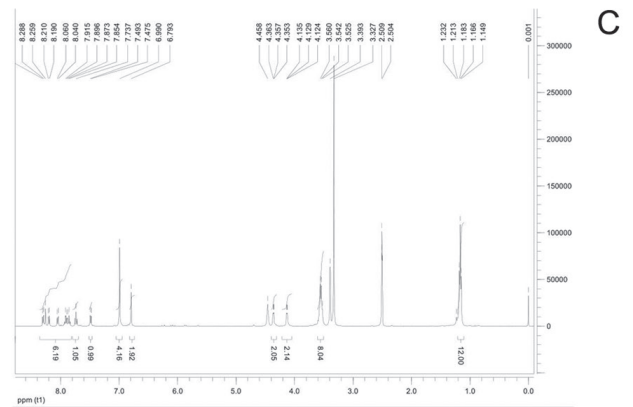

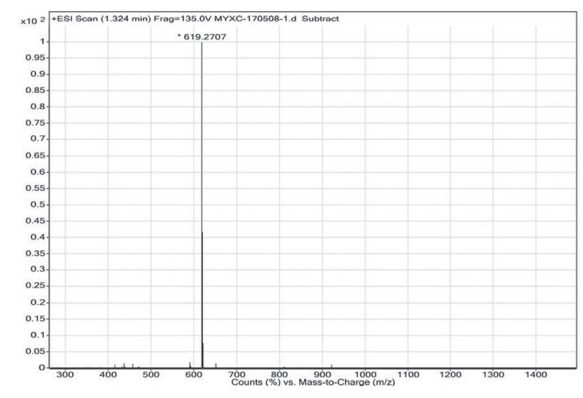

Figure 1. Synthesis pathway of probe. (A) The compound S1-4 (also known as V) was synthesized using the synthesis pathway. The structure of probe was identified by (B) ${ }^{1} \mathrm{H}$-nuclear magnetic resonance spectroscopy and (C) electron ionization-high resolution mass spectrometry.

(ROC) curve was produced to compare the accuracy, specificity and sensitivity of probe in biological samples.

Statistical analysis. Data are represented as mean $\pm \mathrm{SD}$ and were analyzed using SPSS 11.0 software (SPSS, Inc.). One-way ANOVA and the Tukey post hoc test was used for general measurement data. Pearson's correlation coefficient was used for correlative analysis. ROC curve was calculated using GraphPad Prism 7.0 (GraphPad Software, Inc.). P<0.05 was considered to indicate a statistically significant difference.

\section{Results}

The structural identification of the probe. To illustrate the structure of the probe, the following results were obtained using ${ }^{1} \mathrm{H}-\mathrm{NMR}$ and EI-HRMS spectroscopy: ${ }^{1} \mathrm{H}$ NMR (400 MHz, DMSO-D6) $\delta 10.07$ (s, 1H, -CHO), 8.30-7.90 (m, 6H), 7.74 (t, J=7.6 Hz, 1H), 7.49 (d, J=7.2 Hz, 1H), 6.99 (m, 4H), 6.79 (s, 2H), 4.35 (t, J=2.4Hz, 2H), 4.13 (t, J=2.4 Hz, 2H), 3.55 (q, J=7.2 Hz, 8H), 1.17 (t, J=6.8 Hz, 12H; Fig. 1B); EI-HRMS $\left(\mathrm{C}_{38} \mathrm{H}_{39} \mathrm{~N}_{2} \mathrm{O}_{6}{ }^{+}\right.$; Fig. 1C). Molecular weight: 619.2707. Thus, S1-4 was identified as the target compound.

Linearity of Hcy probe detection. Following probe synthesis, to explore the correlation between fluorescence intensity and Hcy concentration, the fluorescence intensity of the S1-4 probe in the presence of ascending concentrations of Hcy was measured. A plot displaying fluorescence intensity of the probe vs. Hcy concentration was obtained (Fig. 2). At the concentration range of $0-100 \mu \mathrm{M}$, fluorescence intensity of the probe

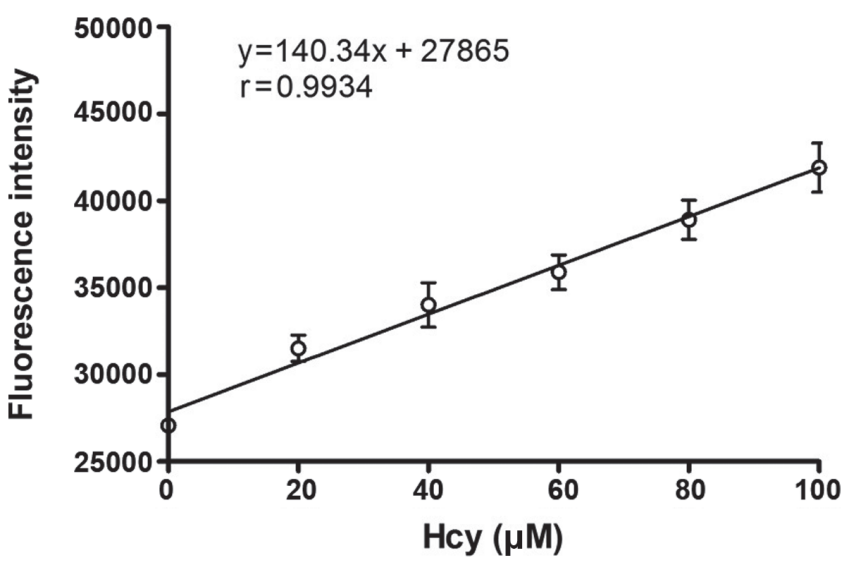

Figure 2. Linearity of the detection of Hcy by the probe. The graph demonstrates that there was a positive correlation between fluorescence intensity of the probe and Hcy concentration in the $0-100 \mu \mathrm{M}$ range. Hcy, homocysteine.

exhibited a good linear relationship with Hcy concentration $(r=0.9934)$.

Effect of fluid viscosity and $\mathrm{pH}$ on the responsiveness of the probe. Hcy at a concentration of $20 \mu \mathrm{M}$ was selected as it is the concentration found in the majority of healthy individuals (8). To characterize the probe under physiological conditions in the human bloodstream (including blood, $\mathrm{pH}$ and viscosity), following reaction with $20 \mu \mathrm{M} \mathrm{Hcy}$, the fluorescence intensity of the S1-4 probe did not appear to be affected by the increasing viscosities of the reaction mixture (glycerol/water, 1.005-219 cp; 

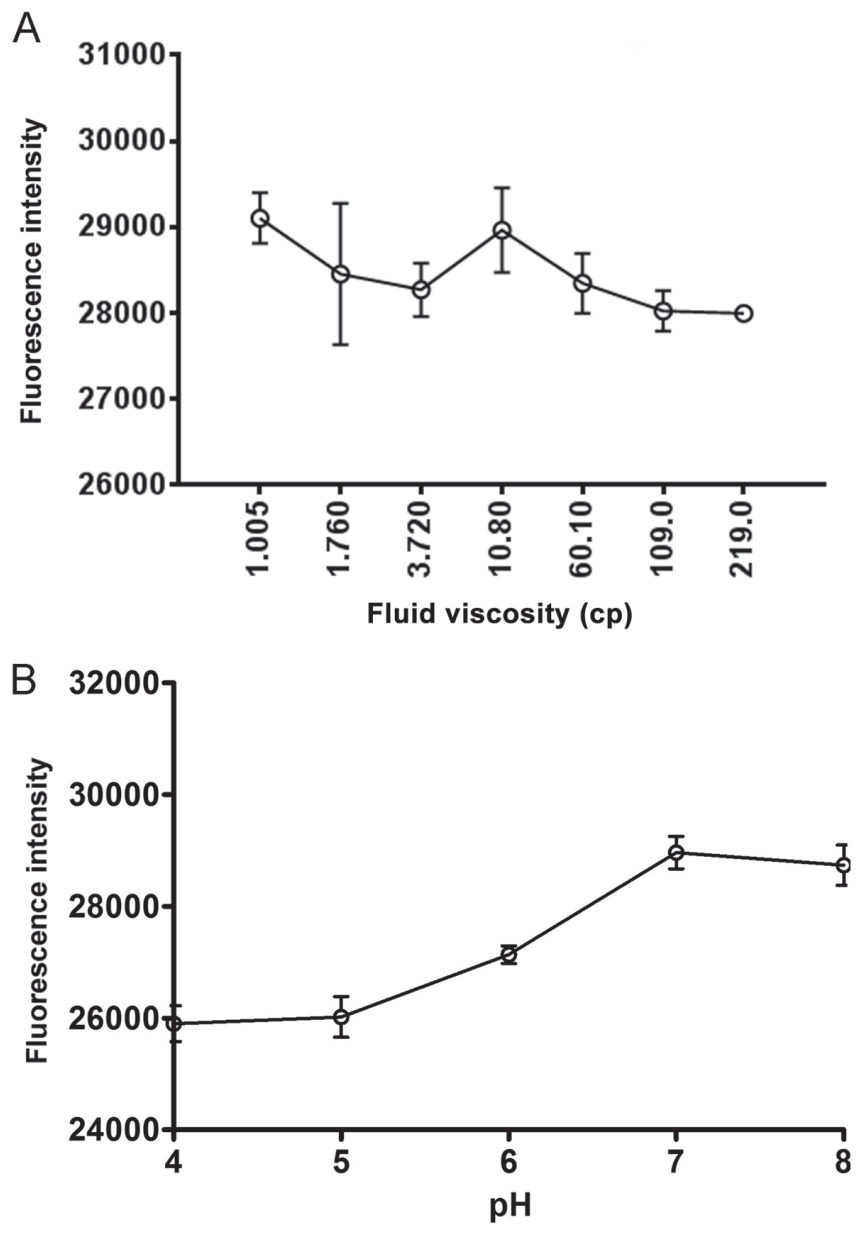

Figure 3. Effects of fluid viscosity and $\mathrm{pH}$ on the responsiveness of the Hcy probe. (A) The graph demonstrates that fluorescence intensity of the probe in response to Hcy was not affected by the different viscosities (glycerol/water, 1.005-219 cp). (B) The graph demonstrates that the fluorescence intensity exhibited stable responsiveness to Hcy $(20 \mu \mathrm{M})$ at $\mathrm{pH} 7$. Hcy, homocysteine.

Fig. 3A). The viscosity range was selected as it spans the normal and abnormally high viscosities observed in healthy and patients with diseases, respectively (9). This suggested that probe activity is unlikely to be affected by changes in blood viscosity induced by changes to physiological functions or diseases (Fig. 4A). The probe exhibited the most fluorescence intensity (responsiveness) to Hcy at $\mathrm{pH} 7$, which suggested that the activity of the probe is unlikely to be affected by changes in blood $\mathrm{pH}$ induced by changes to physiological functions or diseases (Fig. 3B).

Selectivity of the probe in the presence of different biological analytes. None of the interfering substances tested, including amino acids and high concentrations of various metal ions (see Materials and methods), appeared to have adversely affected the fluorescence intensity of the S1-4 probe in response to $20 \mu \mathrm{M}$ Hcy (Fig. 4). These observations suggested that the responsiveness of the probe has good selectivity for Hcy as the fluorescence intensity of Hcy was significant greater compared with cysteine and glutathione.

Measurement of Hcy in human serum. The probe was used to determine if it was able measure serum Hcy levels in healthy people, in addition to patients in the TCI and CI groups. The levels of Hcy between the three groups could be distinguished by the probe. The serum levels of Hcy, as measured by the probe, was significantly higher in the CI group compared with the Norm group (Fig. 5A) and correlated positively with corresponding biochemistry data as measured using a Biochemistry Analyzer (Fig. 5B). However, according to the ROC analysis, the specificity and sensitivity of the S1-4 probe was the same as the analysis by the Biochemistry Analyzer, which suggested that the S1-4 probe needs to be optimized in further studies in the CI group (Fig. 5C and D).

\section{Discussion}

Hcy is a sulfur-containing amino acid that cannot be synthesized in vivo and can only be produced by the catabolism of methionine (10). Under normal circumstances, the Hcy content in the blood is very low, and high concentrations of Hcy have been found to be an independent risk factor for coronary artery disease, cerebrovascular disease and peripheral vascular disease; the risk of developing these conditions are increased with the increase in Hcy concentration (11-14). Some developed and developing countries, including China, the United States and Japan have large and rapidly aging populations. Hcy is an important diagnostic marker for cerebral infarction (15); therefore, it is of great importance to develop an effective Hcy detection method with potentially beneficial values in clinical applications.

Existing Hcy detection methods, such as enzymatic methods, high performance liquid chromatography, capillary electrophoresis separation and immunoturbidimetry, all entail high detection costs and produce ambiguous data, because Hcy, GSH and Cys compounds all contain a sulfhydryl group. Therefore, new Hcy detection technologies that are accurate and efficient are an urgent need in the clinical setting.

In the present study, Rhodamine B was used as the parent compound, and a new compound that has not been previously reported was synthesized. It was found that the probe could selectively react with the amino group and the thiol group in Hcy. It was hypothesized that the fluorescent probe could measure the levels of Hcy and may be applied as a potential clinical detection method for cerebral infarction. The authors then found, that the spatial structure of S1-4 caused a change in the fluorescence intensity of the system, effectively reflecting the level of Hcy in vitro. Therefore S1-4 was researched as a priority in the present study. From the fluorescence intensity measurements, it was effectively demonstrated that the probe can enhance the fluorescence intensity of the reaction media with Hcy. This meant that Hcy could be selectively detected without off-target responses to cysteine and other amino acids, resulting in good selectivity.

Significant structural homology of the small molecule sulfhydryl-containing biological compounds meant that few studies have been able to report the direct detection of various sulfhydryl compounds (such as Cys and GSH) using small molecule fluorescent probes (16). The probe reported in the present study exhibited good water solubility and linear relationship with Hcy concentration, and also demonstrated good selectivity even in the presence of common amino acid and ions found in serum. Importantly, it could discriminate Hcy from other sulfhydryl compounds such as Cys and GSH, potentially avoiding off-target interference. 


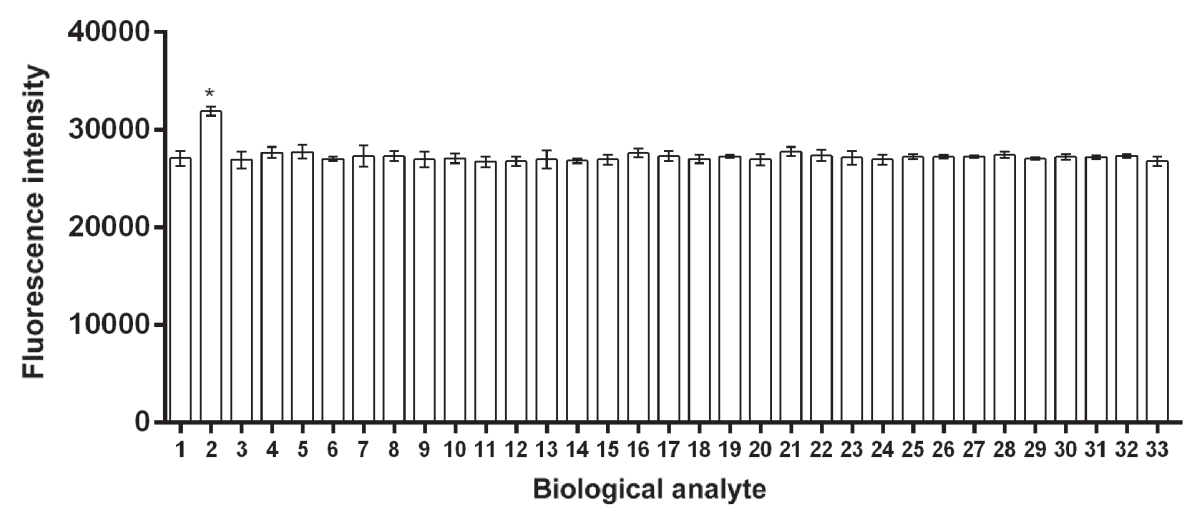

Figure 4. Selectivity of the probe in the presence of different biological analytes. The graph illustrates that there was a significantly increased fluorescence intensity of the probe in response to Hcy compared with other biological analytes. "P<0.05 vs. the other biological analytes. 1, cysteine; 2 , Hcy; 3 , $\mathrm{N}$-acetylcysteine; 4, glutathione; 5 , arginine; 6 , asparagine; 7 , aspartic acid; $8, \beta$-alanine; 9 , dithiothreitol; 10 , glutamine; 11 , glutamic acid; 12 , glycine; 13 , histidine; 14, isoleucine; 15 , L-alanine; 16, leucine; 17, lysine; 18, methionine; 19, phenylalanine; 20, proline; 21, serine; 22, threonine; 23, tryptophan; 24, tyrosine; 25 , valine; 26 , potassium $\left(\mathrm{K}^{+}\right) ; 27$, sodium $\left(\mathrm{Na}^{+}\right) ; 28$, calcium $\left(\mathrm{Ca}^{2+}\right) ; 29$, magnesium $\left(\mathrm{Mg}^{2+}\right) ; 30$, zinc $\left(\mathrm{Zn}^{2+}\right) ; 31$, aluminum $\left(\mathrm{Al}^{3+}\right) ; 32$, cadmium $\left(\mathrm{Cd}^{2+}\right)$; 33, manganese $\left(\mathrm{Mn}^{2+}\right)$. Hcy, homocysteine.
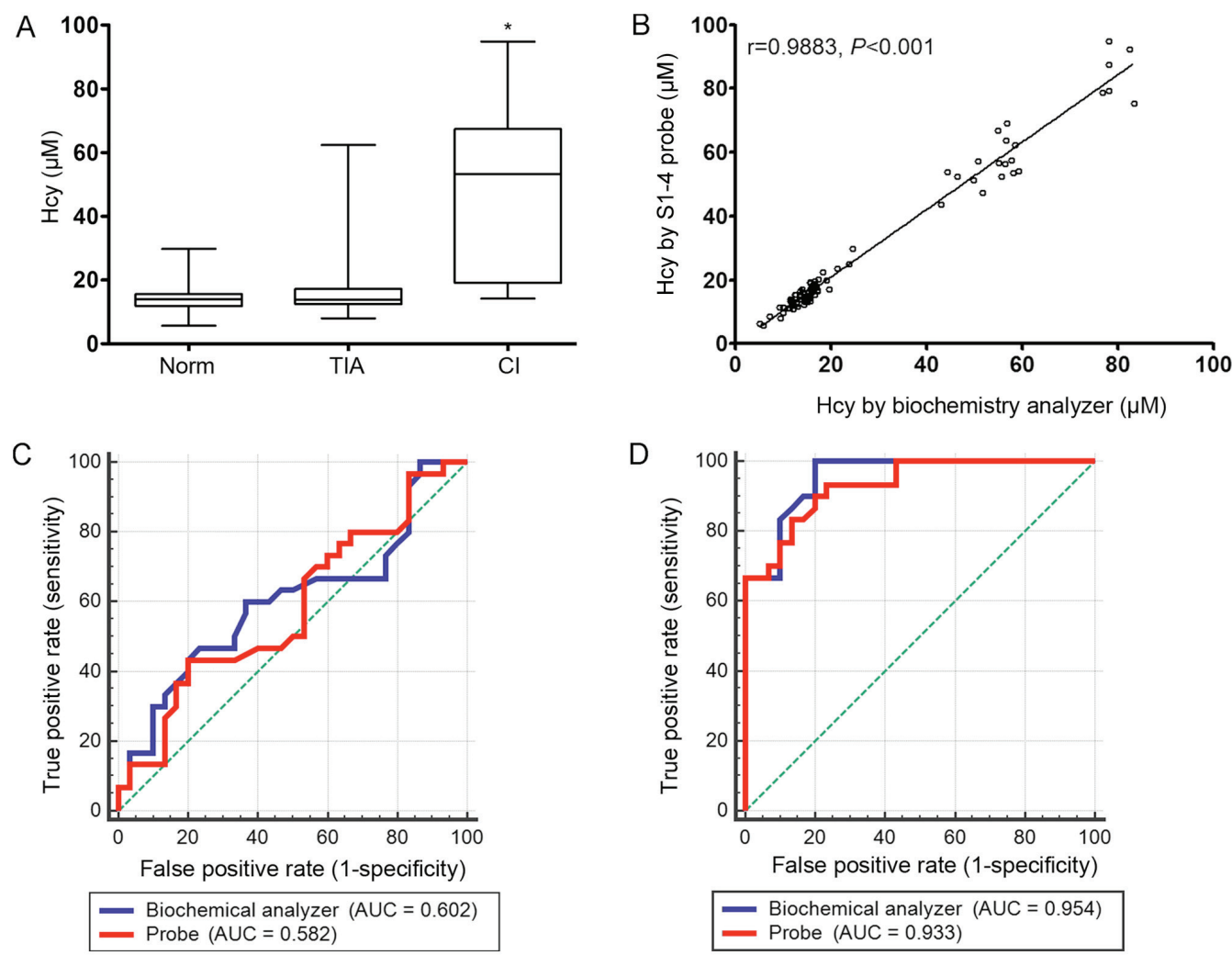

Figure. 5. Measurement of Hcy in human serum. (A) The graph demonstrates that there was a significantly increased level of Hcy in patients in the CI group compared with patients in the Norm and TIA groups. "P<0.05 vs. CI group. (B) The graph illustrates that there was a significant positive correlation between the levels of Hcy as measured using biochemistry and the probe method in the three groups. (C) The ROC analysis displays the specificity and sensitivity of the probe in the TIA group compared with the Biochemistry Analyzer. (D) The ROC analysis displays the specificity and sensitivity of the probe in the CI group compared with the biochemistry analyzer. CI, cerebral infarction; Hcy, homocysteine; Norm, normal; ROC, receiver operating characteristic; TIA, transient cerebral ischemia.

Clinically, this probe also effectively reflected the level of Hcy in serum of healthy people, as well as patients with TCI and CI. The measurements from clinical specimens demonstrated that the fluorescence signal of the probes in patients with CI was significantly higher compared with those in the healthy control group and in the TIA group; which were comparable with those obtained from the Biochemistry Analyzer. This result supported the notion that this probe may have potential clinical application value.

In a recent study, Yang et al (17) reported a near-infrared fluorescent probe for Cys and Hcy detection using the colorimetry method. However, the probe proposed by that study 
is suitable for monitoring and visualizing Cys and Hcy in cells, whereas the probe synthesized by the present study is only suitable for monitoring Hcy in blood sera. In addition, ROC curve analysis reported unsatisfactory probe specificity and sensitivity compared with data from the Biochemistry Analyzer, which indicated a need to optimize this probe for further study, such as by improving the chemical structure and linking to better vehicles. In the future, the number of specimens will need to be increased further, with the detection results compared with those obtained using existing established detection methods to develop a new type of Hcy detection probe. As diabetes mellitus, fatty liver disease and other metabolic diseases are also risk factors for cerebral infarction, the efficacy of the S1-4 probe in measurements of Hcy levels in patients with these diseases will also be explored further.

\section{Acknowledgements}

Not applicable.

\section{Funding}

The present study was supported by Tianjin Science and Technology Key Project on Chronic Diseases Prevention and Treatment (grant no. 16ZXMJSY-00020), Tianjin Municipal Special Program of Talents Development for Excellent Youth Scholars, China (grant no. TJTZJH-QNBJRC-2-9), Tianjin 131 Creative Talents Cultivation Project (1st Class, 2016).

\section{Availability of data and materials}

All data generated or analyzed during the present study are included in this published article.

\section{Authors' contributions}

$\mathrm{YZ}$ and $\mathrm{YM}$ conceived and designed the study. $\mathrm{XZ}$ acquired the data. XL performed the data analysis.

\section{Ethics approval and consent to participate}

This study was approved by the medical ethics committee of Tianjin Huanhu Hospital (Tianjin, China), in accordance with the Declaration of Helsinki; all patients provided informed consent prior to enrollment in the study.

\section{Patient consent for publication}

Not applicable.

\section{Competing interests}

The authors declare that they have no competing interests.

\section{References}

1. Zhong C, Xu T, Xu T, Peng Y, Wang A, Wang J, Peng H, Li Q, Geng D, Zhang D, et al: Plasma homocysteine and prognosis of acute ischemic stroke: A gender-specific analysis from CATIS randomized clinical trial. Mol Neurobiol 54: 2022-2030, 2017.

2. Liu J, Zhu Y, Wu Y, Liu Y, Teng Z and Hao Y: Association of carotid atherosclerosis and recurrent cerebral infarction in the Chinese population: A meta-analysis. Neuropsychiatr Dis Treat 13: 527-533, 2017.

3. Sun LC, Chen R, Fu C, Chen Y, Wu Q, Chen R, Lin X and Luo S: Efficacy and safety of botulinum toxin type a for limb spasticity after stroke: A meta-analysis of randomized controlled trials. Biomed Res Int 2019: 8329306, 2019.

4. Tsai CF, Anderson N, Thomas B and Sudlow CL: Comparing risk factor profiles between intracerebral hemorrhage and ischemic stroke in Chinese and white populations: Systematic review and meta-analysis. PLoS One 11: e0151743, 2016.

5. Wu W, Guan Y, Xu K, Fu XJ, Lei XF, Lei LJ, Zhang ZQ, Cheng Y and Li YQ: Plasma homocysteine levels predict the risk of acute cerebral infarction in patients with carotid artery lesions. Mol Neurobiol 54: 2510-2517, 2016.

6. Fox CK, Mackay MT, Dowling MM, Pergami P, Titomanlio L and Deveber G; SIPS Investigators: Prolonged or recurrent acute seizures after pediatric arterial ischemic stroke are associated with increasing epilepsy risk. Dev Med Child Neurol 59: 38-44, 2017.

7. Cioni G, Marcucci R, Gori AM, Valente S, Giglioli C, Gensini GF, Abbate R and Boddi M: Increased homocysteine and lipoprotein(a) levels highlight systemic atherosclerotic burden in patients with a history of acute coronary syndromes. J Vasc Surg 64: 163-170, 2016.

8. Kumar M and Sandhir R: Neuroprotective effect of hydrogen sulfide in hyperhomocysteinemia is mediated through antioxidant action involving Nrf2. Neuromolecular Med 20: 475-490, 2018.

9. Senturk B, Akdeniz B, Yilmaz MB, Ozcan Kahraman B, Acar B Uslu $S$ and Birlik M: Whole blood viscosity in systemic sclerosis: A potential biomarker of pulmonary hypertension? Clin Rheumatol, May 26, 2019 (Epub ahead of print).

10. Ling-Cong, Hong-Zhao, Yu-Wang, Yu-Li and Xin-Sui: The serum homocysteine level in patients with acute ischemic stroke (AIS) after thrombolysis and its relationship with clinical outcomes. Rev Assoc Med Bras (1992) 64: 438-442, 2018.

11. Wu X, Zhang L, Miao Y, Yang J, Wang X, Wang CC, Feng J and Wang L: Homocysteine causes vascular endothelial dysfunction by disrupting endoplasmic reticulum redox homeostasis. Redox Biol 20: 46-59, 2019.

12. Kim J, Pyo S, Yoon DW, Lee S, Lim JY, Heo JS, Lee S and Shin C: The co-existence of elevated high sensitivity C-reactive protein and homocysteine levels is associated with increased risk of metabolic syndrome: A 6-year follow-up study. PLoS One 13: e0206157, 2018.

13. Hu H, Wang C, Jin Y, Meng Q, Liu Q, Liu Z, Liu K, Liu X and Sun H: Catalpol inhibits homocysteine-induced oxidation and inflammation via inhibiting Nox4/NF- $\mathrm{BB}$ and GRP78/PERK pathways in human aorta endothelial cells. Inflammation 42: 64-80, 2019.

14. Saoji R, Das RS, Desai M, Pasi A, Sachdeva G, Das TK and Khatkhatay MI: Association of high-density lipoprotein, triglycerides, and homocysteine with bone mineral density in young Indian tribal women. Arch Osteoporos 13: 108, 2018.

15. Liu J, Quan J, Li Y, Wu Y and Yang L: Blood homocysteine levels could predict major adverse cardiac events in patients with acute coronary syndrome: A STROBE-compliant observational study. Medicine (Baltimore) 97: e12626, 2018.

16. Zhang W, Liu J, Yu Y, Han Q, Cheng T, Shen J, Wang B and Jiang Y: A novel near-infrared fluorescent probe for highly selective detection of cysteine and its application in living cells. Talanta 185: 477-482, 2018.

17. Yang $\mathrm{X}$, Wang $\mathrm{Y}$, Zhao MX and Yang W: A colorimetric and near-infrared fluorescent probe for cysteine and homocysteine detection. Spectrochim Acta A Mol Biomol Spectrosc 212: 10-14, 2019. 